\title{
KY electroproduction at CLAS12
}

\author{
Lucilla Lanza (for the CLAS Collaboration) $)^{1, *}$ \\ ${ }^{1}$ INFN, Istituto Nazionale di Fisica Nucleare, Sezione di Roma Tor Vergata
}

\begin{abstract}
An experimental program has been approved at the Thomas Jefferson National Accelerator Facility to measure the $\left(\mathrm{ep}, \mathrm{e}^{\prime} \mathrm{K}^{+}\right) \mathrm{Y}$ reactions using the CLAS12 setup in Hall B. Data have been obtained using a 7.5 $\mathrm{GeV}$ electron beam, impinging upon a liquid hydrogen target in the CLAS12 center. Scattered electrons have been detected in an angle range of $2.5^{\circ}$ to $4.5^{\circ}$ by the Forward Tagger (FT) and at angles greater than $5^{\circ}$ in the CLAS12 Forward Detector, allowing to measure the KY electroproduction differential cross section and to probe the $Q^{2}$ evolution of the $\mathrm{N}^{*}$ resonances electrocouplings in the $Q^{2}$ range from $0.05 \mathrm{GeV}^{2}$ to $3 \mathrm{GeV}^{2}$. The study of the $Q^{2}$ dependence of the electrocouplings will provide a crucial tool to investigate the possible hybrid nature of the $\mathrm{N}^{*}$ resonances. Preliminary results from CLAS12 data are compared with simulated data using a realistic Gent Regge plus resonance event generator.
\end{abstract}

\section{Introduction}

Besides regular mesons and baryons a number of "exotic" hadrons have been conjectured, states having more valence quarks than the three quark configuration for baryons or the quark-antiquark one for mesons, namely tetraquarks and pentaquarks, or states having valence gluons in their structure, hybrid mesons and hybrid baryons.

Hybrid baryons are hypothetical three quark states with gluonic degrees of freedom. Their existence is allowed by Quantum Chromo Dynamics (QCD), and Lattice QCD (LQCD) calculations predict the existence of several hybrid states $1.3 \mathrm{GeV}$ above the nucleon ground state [1].

The search for hybrid baryons is more difficult than for hybrid mesons because of issues in distinguishing a hybrid from a regular baryon. In fact, differently from hybrid mesons, hybrid and regular baryons can have the same quantum numbers. A useful approach has been recently employed in the study of the Roper resonance $\mathrm{N}(1440) 1 / 2^{+}$, that has been considered as a candidate for the lowest mass hybrid baryon [2]. To dismiss this possibility [3] [4] [5], meson electroproduction data were exploited, studying the photon virtuality $\left(Q^{2}\right)$ evolution of the electrocouplings, that should present a different low $Q^{2}$ behavior for hybrids with respect to regular states. The ongoing program at the Thomas Jefferson National Accelerator Facility (Jefferson Laboratory) in Newport News (VA) employs photoproduction and electroproduction reactions to study the excitation of nucleons in the nucleon resonance mass range up to $3 \mathrm{GeV}$ and proved to be successful; moreover a program concerning the search for hybrid baryons is in progress.

The task is to perform an extensive study of $K^{+} \Lambda, K^{+} \Sigma$ and $p \pi^{+} \pi^{-}$electroproduction reactions, in the kinematic region that covers the mass range up to $3 \mathrm{GeV}$ and in the $Q^{2}$ range

*e-mail: lucilla.lanza@roma2.infn.it from $0.05 \mathrm{GeV}^{2}$ to $2.0 \mathrm{GeV}^{2}$ with CLAS12 experimental setup. Once the existence of a new baryonic resonance is assessed, it will be possible to establish whether it is a hybrid or not by studying the $Q^{2}$ evolution of its electrocouplings. Data taking has started during Fall 2018 and the first set of data is at present under analysis. ${ }^{1}$

\section{Theoretical background}

First Lattice QCD predictions regarding hybrids were formulated in 2003 [6]. These calculations used the quenched approximation, i.e. the quantum loops of fermions in Feynman diagrams were neglected. The lowest hybrid baryon mass was predicted around $2 \mathrm{GeV}, 1 \mathrm{GeV}$ above the nucleon mass. In 2012 the first unquenched LQCD calculations were proposed [1]. Figure 1 shows the extracted spectrum of Nucleon and Delta states in the lowest mass range. The $\mathrm{N}^{*}$ and $\Delta^{*}$ denote excited states of the Nucleon and Delta, respectively. Grey boxes are regular states and blue boxes are states identified as hybrids. The corresponding negative parity hybrid states are predicted to be heavier.

The first group of hybrid baryons is expected to lie in a restricted energy band of $\sim 300 \mathrm{MeV}$ not occupied by ordinary baryons, $1.3 \mathrm{GeV}$ above the nucleon ground state, with $J^{P}=1 / 2^{+}$. If their width is not too broad it should be possible to observe them experimentally.

Besides the mass range prediction that has just been described, the signature to distinguish a hybrid baryon from an ordinary one arises from the study of their electromagnetic couplings which is described in section 2.1.

\footnotetext{
${ }^{1}$ The Hybrid Baryons program has been approved on July 2016 by Jefferson Laboratory Program Advisory Committee (PAC) 44.
} 


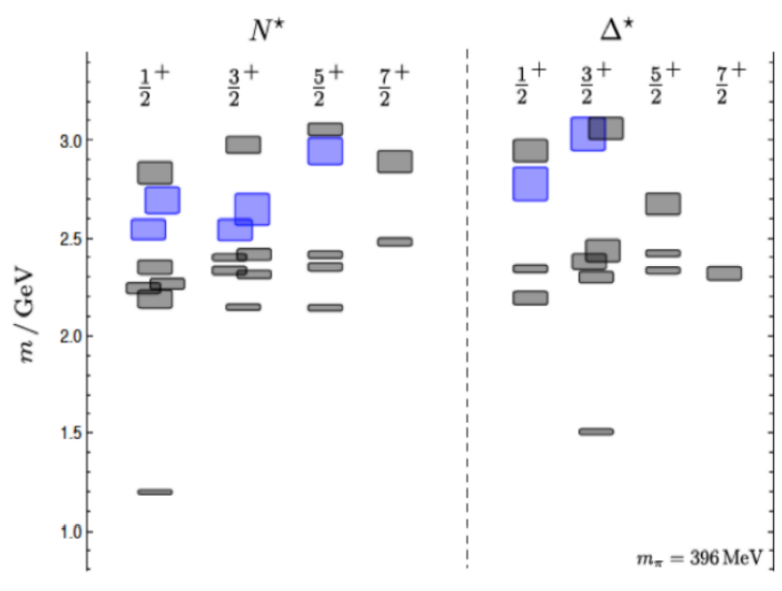

Figure 1. The light-quark baryon spectrum predicted in Lattice QCD at a pion mass of $396 \mathrm{MeV}$ [1]. Grey boxes are conventional qqq states and the blue shaded boxes indicate hybrid baryons states. Note that both the mass of the nucleon ground state and of the $\Delta(1232)$ are shifted by nearly $300 \mathrm{MeV}$ to higher masses.

\subsection{Electromagnetic Couplings}

Hybrid and regular baryons are expected to have the same quantum numbers, but the information from the electrocouplings can be exploited to identify their nature. In fact a distinctively different $Q^{2}$ evolution of the hybrid baryon electrocouplings is expected considering the different color-multiplet assignments for the quark-core in a regular versus a hybrid baryon, i.e. a color singlet versus octet. Theoretical models [2] predict a peculiar behavior of hybrid baryons electrocouplings at low $Q^{2}$ : a fast drop of the transverse helicity amplitude $A_{1 / 2}\left(Q^{2}\right)$ and a suppression of the longitudinal helicity amplitude $S_{1 / 2}\left(Q^{2}\right)$.

Information from electrocouplings evolution with $Q^{2}$ has been exploited to solve the Roper puzzle, see [4] from which figure 2 is extracted. In this figure the full circles are the results obtained in [4] from CLAS data, the bands show the model uncertainties, the open boxes are the results of the combined analysis of CLAS single $\pi$ and $2 \pi$ electroproduction data [7], the full triangle at $Q^{2}=0$ is the Review of Particle Physics (RPP) estimate [8], the thick curves correspond to the results obtained in the light-front relativistic quark models (LF RQM) assuming that $\mathrm{N}(1440) P_{11}$ is a first radial excitation of the 3-quark ground state: [9] (dashed), [10] (solid). Finally the thin dashed curves are obtained assuming that $\mathrm{N}(1440) P_{11}$ is a hybrid baryon [2]. It is evident for both amplitudes that data points are inconsistent with the hybrid predictions, especially in the longitudinal helicity amplitude case, where in the hybrid case a suppression was expected and large values occur instead.

\subsection{Hadronic Couplings}

Unfortunately there is a lack of information regarding possible hadronic couplings of hybrid baryons. From the

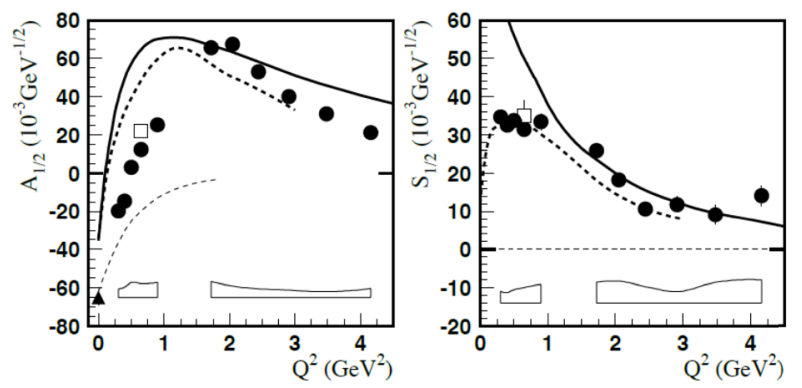

Figure 2. Helicity amplitudes for the $\gamma^{*} p \rightarrow N(1440) P_{11}$ transition. The full circles are the results obtained in [4] from CLAS data. The bands show the model uncertainties. The open boxes are the results of the combined analysis of CLAS single $\pi$ and $2 \pi$ electroproduction data [7]. The full triangle at $Q^{2}=0$ is the Review of Particle Physics (RPP) estimate [8]. The thick curves correspond to the results obtained in the LF relativistic quark models assuming that $\mathrm{N}(1440) P_{11}$ is a first radial excitation of the 3-quark ground state: [9] (dashed), [10] (solid). The thin dashed curves are obtained assuming that $\mathrm{N}(1440) P_{11}$ is a gluonic baryon excitation ( $q^{3} \mathrm{G}$ hybrid state) [2].

knowledge of the hadronic couplings one could select the best channel for an experimental study, but without theoretical information any experimental approach must rely on a set of assumptions. Final states with significant gluonic admixture, e.g. $B_{G} \rightarrow N \eta^{\prime}$ [11] (the G subscript denotes a gluon component), should be preferential as well as final states containing $s \bar{s}$ contributions due to the $B_{G} \rightarrow$ $s \bar{s}$ coupling, e.g. $B_{G} \rightarrow K^{+} \Lambda, B_{G} \rightarrow N^{*}(1535) \pi \rightarrow N \eta \pi$, $B_{G} \rightarrow \phi(1020) N$ and $B_{G} \rightarrow K^{*} \Lambda$, or final states to which the missing resonances are predicted to mainly decay, such as $B_{G} \rightarrow N \pi \pi$. Among all possible candidate final states, the $K^{+} Y$ and the $N \pi \pi$ have been selected to study the sensitivity of the electroproduction reaction to the formation of a hybrid resonance, since they are the easiest channel among the listed ones for which a theoretical description is available. This work is focused on the $K^{+} \Lambda$ channel.

\subsection{Signature for Hybrid Baryon States}

Summarizing our understanding, based on available knowledge, we may state that the signature for the lower mass hybrid baryons may consist of:

- Resonances not predicted by conventional constituent quark models with $\mathrm{I}=\frac{1}{2}, J^{P}=\frac{1}{2}^{+}$and $J^{P}=\frac{3}{2}^{+}$, with masses from $1.8 \mathrm{GeV}$ to $2.5 \mathrm{GeV}$ decaying into $\mathrm{N} \pi \pi$, $K^{+} \Lambda$ or $K^{+} \Sigma^{0}$ final states;

- Drop of the transverse helicity amplitudes $A_{1 / 2}\left(Q^{2}\right)$ and $A_{3 / 2}\left(Q^{2}\right)$ as a function of $Q^{2}$ faster than for ordinary three quark states, because of the extra glue-component in the valence structure;

- Suppressed longitudinal amplitude $S_{1 / 2}\left(Q^{2}\right)$ in comparison to transverse electro-excitation amplitudes. 


\section{CLAS12 and Forward Tagger at Jefferson Laboratory}

The experiment is located at Jefferson Laboratory, in Hall B, with CLAS12 experimental setup [12].

The CLAS12 detector, shown in figure 3, has been used to take data with longitudinally polarized high energy electron beams scattering on a liquid-hydrogen target, with an operating luminosity of $L=10^{35} \mathrm{~cm}^{-2} \mathrm{~s}^{-1}$ which corresponds to a total hadronic rate of $5 \times 10^{6} \mathrm{~s}^{-1}$.

The electron beam has been provided by the CEBAF accelerator with three values of energy in different runs: 6.5 $\mathrm{GeV}, 7.5 \mathrm{GeV}$ and $10.6 \mathrm{GeV}$.

The CLAS12 detector is highly hermetic, provides high

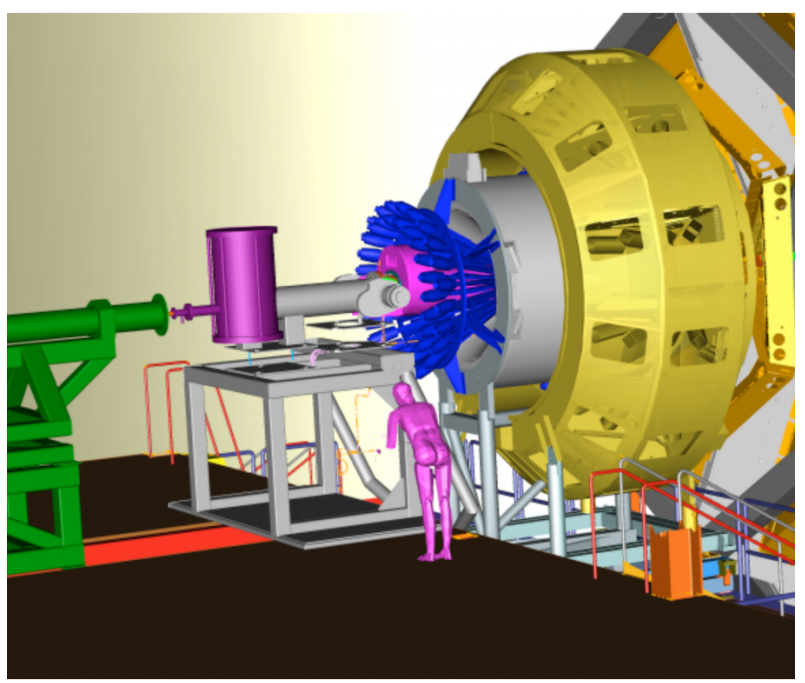

Figure 3. Sketch of the CLAS12 detector [12]: it consists of a central detector $(\mathrm{CD})$ equipped with a superconducting solenoid and a forward detector (FD) equipped with a torus magnet, to cover polar angular ranges from $35^{\circ}$ to $125^{\circ}$ and from $5^{\circ}$ to $35^{\circ}$, respectively.

multiplicity reconstruction, can run at high luminosity and allows to carry out the experiment under study. CLAS12 provides charged particle identification by correlating the particle speed $\beta$, measured by time of flight detectors, with momentum, the curvature of charged particle tracks in the high magnetic field. Cherenkov counters are used for electron-pion separation. CLAS12 consists of a Forward Detector, covering the forward polar angle range between $5^{\circ}$ and $35^{\circ}$, and a Central Detector, covering larger angles up to $125^{\circ}$. The forward angular region is equipped with a torus magnet, while the central polar angle range of $35^{\circ}$ to $125^{\circ}$. The forward angular region is equipped with a torus magnet, while the central polar angle range of $35^{\circ}$ to $125^{\circ}$ is provided with a $5 \mathrm{~T}$ solenoid magnet.

\subsection{Forward Detector}

The Forward Detector is the part of the CLAS12 detector devoted to the measurement and identification of charged and neutral particles scattered between 5 and 35 degrees, in the full azimuthal angle. It consists of six identical magnetic spectrometers working in conjunction with a toroidal field, generated by a six coil superconducting magnet. Each spectrometer, corresponding to a sector of the Forward Detector, is composed of a high threshold Cherenkov counter, a set of drift chambers, a low threshold Cherenkov counter, scintillation counters and an electromagnetic calorimeter. The working principle of the Forward Detector is the following: charged particles are detected from their trajectory due to the torus magnet magnetic field and their momentum is reconstructed using the drift chambers. The Cherenkov counters and the time-offlight detectors provide information useful for the discrimination among particles and the electromagnetic calorimeter gives the information about the energy. The design momentum resolution is $\frac{\sigma_{E}}{E} \simeq \frac{0.1}{\sqrt{E}}$ and the design parameters were chosen to allow for pions-kaons and for protonskaons separation up to $3 \mathrm{GeV} / \mathrm{c}$ and up to $4 \mathrm{GeV} / \mathrm{c}$, respectively.

\subsection{Central Detector}

The Central Detector is located around the target to detect particles emitted at low momentum, $<1.5 \mathrm{GeV} / \mathrm{c}$, with large polar angles, between 35 and 125 degrees, in the full azimuthal range. It is composed of a 5T superconducting solenoid magnet, a Silicon Vertex Tracker, that surrounds the target, a time-of-flight detector and a Central Neutron Detector. Particle momentum is measured using information from the trackers within the strong solenoid magnetic field. Neutral particles may be detected by the Central Neutron Detector.

\subsection{Forward Tagger}

To extend the detection of scattered electrons to the smallest polar angles, CLAS12 includes a Forward Tagger (FT), shown in figure 4; it allows to detect electrons and photons emitted between 2.5 and 4.5 degrees. The design energy resolution is expected to be $2 \% / \sqrt{E(\mathrm{GeV})} \oplus 1 \%$.

The FT is made of three components:

- Electromagnetic Calorimeter (FT-Cal), to measure the energy of the electromagnetic showers produced by electrons and photons and to provide a fast trigger signal;

- Hodoscope (FT-Hodo), to discriminate between electrons and photons that enter in the calorimeter;

- Tracker (FT-Trck), to measure the polar $\theta_{e}$ and azimuthal $\phi_{e}$ scattering angles of the electrons.

The electron beam interacts with the target via the exchange of a virtual photon, whose four momentum $\mathrm{q}=$ $(v, \mathbf{q})$ is the difference between the beam and the scattered electron momenta. It is possible to express the photon virtuality $Q^{2}$ as a function of the electron scattering angle $\theta_{e}$ and of the energy of the final electron $E_{e^{\prime}}$ :

$$
Q^{2}=4 E_{e} E_{e^{\prime}} \sin ^{2} \frac{\theta_{e}}{2} .
$$


Events for which the electron scattering occurs at very small angles, such as the ones detected by FT, also may have a low $Q^{2}$ (the electroproduction at this $Q^{2}$ can be considered for practical purposes photoproduction). By using electron beams of variable incoming energies $E_{e}$ from 6.5 up to $10.6 \mathrm{GeV}$ and detecting the scattered electron in the angular range from $2.5^{\circ}$ to $135^{\circ}$ it is possible to span $Q^{2}$ virtualities between $0.05 \mathrm{GeV}^{2}$ and $5 \mathrm{GeV}^{2}$. The lowest $Q^{2}$ range is reachable using the lowest beam energy and detecting scattered electrons in the FT.

Considering the importance of events at low $Q^{2}$, the FT is therefore a crucial component for this study.

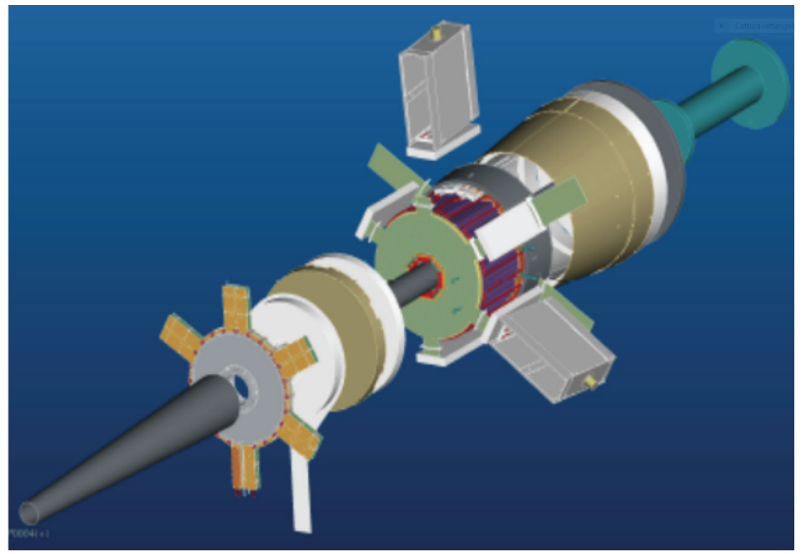

Figure 4. The Forward Tagger system. The FT provides electron and high energy photon detection in a range of polar angles $\theta=$ $2.5^{\circ}-4.5^{\circ}$ and is fully integrated into the operation of CLAS12.

\section{Simulation and Reconstruction of $K^{+} \Lambda$ Electroproduction Events in CLAS12}

To determine the best running conditions for the experiment, simulations have been exploited using an Event Generator based on the Gent RPR-2011 Model [13] to produce electroproduction events and a Monte Carlo to simulate CLAS12 acceptance effects.

\subsection{Regge-Plus-Resonance 2011 Model}

The model employed to describe the KY electroproduction channel is the "Regge-Plus-Resonance" developed by the Gent group. It describes the reaction amplitude as the superposition of tree-level s-channel nucleon resonances on the non-resonant contribution. A set of 11 nucleon resonances has been considered to determine the optimum resonant contribution in the RPR $\mathrm{p}\left(\gamma, K^{+}\right) \Lambda$ framework. The model with the highest Bayesian evidence, called RPR2011, includes the resonances $S_{11}$ (1535), $S_{11}(1650), F_{15}$ (1680), $P_{13}$ (1720), $P_{11}$ (1900), $P_{13}$ (1900), $D_{13}$ (1900) and $F_{15}$ (2000). The non-resonant component is the sum of t-channel exchanges of Reggeized $K^{+}(494)$ and $K^{*+}$ (892).

\subsection{Selection of the Best Running Conditions}

The best running conditions, in terms of beam energy and magnetic field strength, have been determined exploiting simulations.

In order to choose between a positive and a negative value for the torus current, events have been simulated with both the configurations and from the comparison it is possible to notice that the negative one, which corresponds to outbending electrons, provides a smaller acceptance gap between the regions covered by the FT and by CLAS12.

Additionally, by varying the intensity of the torus current in the range between $-1500 \mathrm{~A}$ and $-3375 \mathrm{~A}$, it has been verified that the highest energy resolution obtained with the highest magnetic current configuration provides the best separation between the $K^{+} \Lambda$ and the $K^{+} \Sigma$ processes. Finally, to select the best energy for the electron beam the efficiency curve (ratio between the number of detected events and the generated ones as a function of $Q^{2}$ ) has been studied for three different configurations, $E_{\text {beam }}=6.5 \mathrm{GeV}$, $E_{\text {beam }}=7.5 \mathrm{GeV}$ and $E_{\text {beam }}=10.6 \mathrm{GeV}$. In all three cases the efficiency curve presents a drop between the regions covered by the Forward Tagger apparatus and CLAS12 detector, occurring in correspondence of different $Q^{2}$ ranges. Therefore the information from events taken with the three different beam energies allows to access complementary $Q^{2}$ ranges.

\subsection{CLAS12 Acceptance Effects}

Simulations performed employed a fast Monte Carlo program FASTMC that has been developed to estimate CLAS12 acceptance and event production rates. This code contains geometrical acceptances and parametrized resolution functions for the tracking systems, the electromagnetic calorimeters, the scintillation counters and the Forward Tagger. It also uses realistic field maps for the CLAS12 torus and solenoid, that can be scaled to study the effect of different magnet currents and polarities. The sensitive volumes of the tracking and detection regions have been designed to closely match the CLAS12 detector design. The high efficiency fiducial areas of the detector have been taken into account in the event reconstruction, which also provides a smearing of the final state charged particle momenta.

A full GEANT-based Monte Carlo and reconstruction software code has been developed in the meanwhile for CLAS12, called CLARA. Comparisons of the CLAS12 response using the FASTMC code suite and the full CLAS12 GEANT-4 code suite have shown reasonably good correspondence.

\section{Preliminary Results from Physics Runs}

Physics Runs for CLAS12 at Jefferson Laboratory started in February 2018. The first dedicated Run took data during Fall 2018 for the study of KY channels. Figure 5 shows the kaon missing mass spectrum, $\operatorname{MM}\left(e^{-} K^{+}\right)$, for 15 runs using a $7.5 \mathrm{GeV}$ energy beam on a $5 \mathrm{~cm}$ long liquid-hydrogen target. This amounts to roughly $10 \%$ of 
the dedicated Run Fall $20187.5 \mathrm{GeV}$ data set. The electron is detected in the Forward Electromagnetic Calorimeter and the $K^{+}$is detected in the Forward Detector.

Peaks are visible for $\Lambda(1116), \Sigma(1193), \Sigma(1385), \Lambda(1405)$ and $\Lambda(1520)$. In particular it is possible to distinguish the two peaks of $\Lambda(1116)$ and $\Sigma(1193)$. Events with the electron detected by Forward Tagger are at present under analysis, using the same mass range: while the missing mass resolution is expected to improve after momentum corrections and detector alignment optimization are applied, it is already sufficient to identify the reactions events.

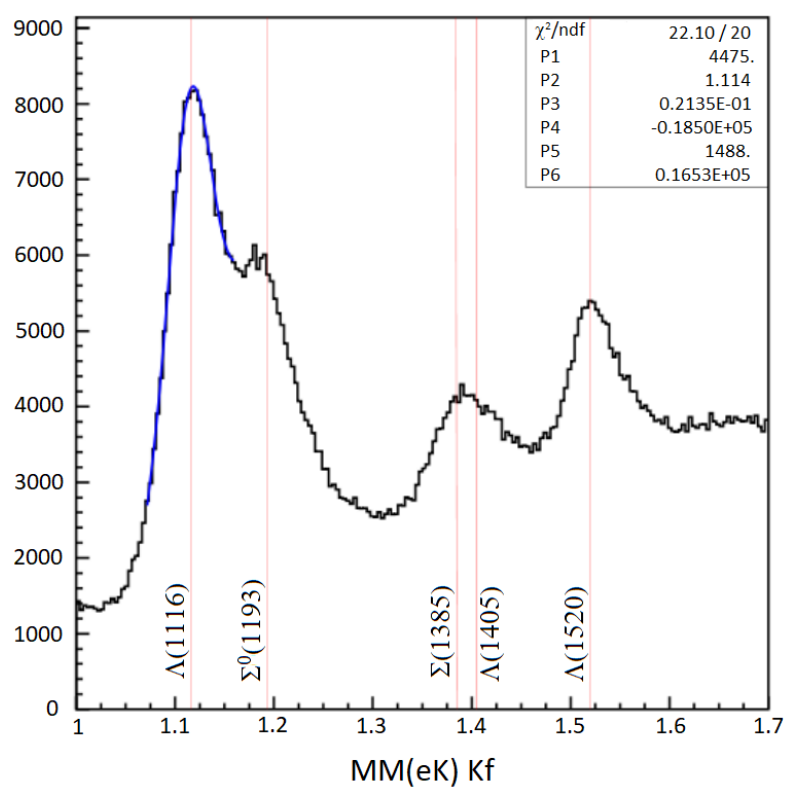

Figure 5. $\mathrm{MM}\left(e^{-} K^{+}\right)(\mathrm{GeV})$ plot using data from 15 Runs acquired during Fall 2018 requiring a $K^{+}$detected in the Forward Detector. The vertical lines on the plot represent the nominal masses of the $\Lambda$ and $\Sigma$ hyperons. The blue line is the fit on the $\Lambda^{0}$ peak and the parameters are in the top right box. $\Lambda^{0}(1116)$ and $\Sigma^{0}(1193)$ baryons peaks can be distinguished. Courtesy of D. Carman.

\section{Conclusions}

This contribution reports preliminary results and analysis for KY electroproduction channels available from a subset of data collected by CLAS12 detector during Fall 2018 at Thomas Jefferson National Accelerator Facility. $\operatorname{MM}\left(e^{-} K^{+}\right)$plots obtained for events with the electron detected by CLAS 12 and the $K^{+}$detected in the Forward Detector present a clear separation between $\Lambda$ and $\Sigma^{0}$ peaks. Next step will be to exploit new calibrated data that will be available soon. These data will be subjected to a full amplitude partial wave analysis, to study the resonance contribution to the KY electroproduction reactions.

\section{References}

[1] J. J. Dudek and R. G. Edwards, Phys Rev. D 85, 054016 (2012)

[2] Z. P. Li and V. Burkert and Z. J. Li, Phys. Rev. D 46, 70 (1992)

[3] I. G. Aznauryan et al. (CLAS Collaboration), Phys. Rev. C 78, 045209 (2008)

[4] I. G. Aznauryan et al. (CLAS Collaboration), Phys. Rev. C 80, 055203 (2009)

[5] I. G. Aznauryan and V. D. Burkert, Prog. Part. Nucl. Phys. 1, 1-54 (2012)

[6] T. T. Takahashi and H. Suganuma, Phys. Rev. Lett. 90, 182001 (2003)

[7] I. G. Aznauryan and V. D. Burkert et al., Phys. Rev. C 72, 045201 (2005)

[8] Amsler et al. (Particle Data Group), Phys. Lett. B 667, $1-6(2008)$

[9] S. Capstick and B. D. Keister, Phys. Rev. D 51, 3598 (1995)

[10] I. G. Aznauryan, Phys. Rev. C 76, 025212 (2007)

[11] E. Kou, Phys. Rev. D 63, 054027 (2001)

[12] CLAS Collaboration, Tech. rep. Jefferson Laboratory (2008)

[13] L. De Cruz and J. Ryckebusch and T. Vrancx and P. Vancraeyveld, Phys. Rev. C 86, 015212 (2012) 